\title{
Discovering a sense of difference was the main experience of growing up with cystic fibrosis
}

D'Auria JP, Christian BJ, Richardson LF. Through the looking glass: children's perceptions of growing up with cystic fibrosis. Can J Nurs Res 1997 Winter;29:99-112.

\section{Question}

How do children with cystic fibrosis (CF) perceive the unfolding of the chronic illness experience?

\section{Design}

Grounded theory.

\section{Setting}

Regional CF centre in south east US.

\section{Patients}

20 children who were 6-12 years of age (mean age 9 y, $60 \%$ boys) and had been diagnosed with CF by 3 years of age.

\section{Methods}

In 30-60 minute audiotaped interviews, children were asked to visualise their experiences of growing up with CF. They were asked to describe their earliest memory and to give advice to a child newly diagnosed with CF. Field notes documented personal characteristics, emotional response, and data collection conditions. Interviews were transcribed, coded, and analysed using the constant comparison method.

\section{Main results}

The central phenomenon of growing up with $\mathrm{CF}$ was discovering a sense of difference. 4 main themes emerged from children's accounts. Puzzling out the meaning described many children's memories of learning from their mothers that they had CF and the surprise they felt at not being like other children. For many children with CF, entering school and exposure to peers began their discovery that they were different; these children were surprised by the response of their peers to their symptoms. Lack of understanding regarding the causality of $\mathrm{CF}$ frequently led younger children (6-9 y of age) to the misconception that their peers avoided them because CF was contagious. Being teased and picked on was a main theme for children with CF. At a time when peer acceptance was crucial, children tended to view themselves through the eyes of their peers and often hid their visible differences so that they could make friends. With time, children learnt to view themselves as normal and to be less affected by the reactions of their peers. Telling others about their illness was another main theme. To avoid the negative social consequences of their visible symptoms and to control how other children viewed them, children with CF kept secrets or disclosed their illness selectively to people whom they considered to be trustworthy. When asked what they would tell younger children with CF about growing up, these children frequently advised them to keep secrets about their illness. Keeping up was a major concern for children with CF. The symptoms and functional limitations of CF highlighted physical differences and made it difficult to fit in and compete with peers. The most restrictive problem for children with $\mathrm{CF}$ was not being able to run as fast as their friends. Many children withdrew and isolated themselves after being teased about their symptoms, which further reduced their opportunity to participate in peer activities. Other children with CF learnt to pace themselves to control their symptoms and minimise their differences from other children.

\section{Conclusions}

Children with cystic fibrosis described discovering a sense of difference when they were asked to talk about what it was like to grow up with a chronic illness. They described being teased and picked on by other children and controlling the image they offered to peers.

Source of funding: in part, National Institute for Nursing Research.

For correspondence:Dr JP D'Auria, School of Nursing, University of North Carolina at Chapel Hill, CB \#7460, Carrington Hall, Chapel Hill, NC 27599-7460, USA. Fax +1 9199667298.

\section{Commentary}

The study by D'Auria et al is an important contribution to the literature because of its focus on the young child's experience of $\mathrm{CF}$, rather than the more traditional focus on the parent or caregiver. The central phenomenon described by the children was that of discovering a sense of difference. Themes identified focused on components of the difference and their effects on the development of peer relationships. These findings are echoed in previous research. Mulderij, in a review of literature and summary of field observations, reported that chronically ill children frequently felt excluded from peer relationships because of factors such as poor social skills, altered physical ability, and frequent interruptions to activities because of therapeutic regimens. ${ }^{1}$

Although analysis in this investigation was limited to children's experiences with CF, a subset of information was obtained about their coping strategies (eg, keeping secrets and how to choose friends). Further analysis of these data would have provided an added dimension to the understanding of how children manage their growing up with CF.

The findings of D'Auria et al are clinically relevant. Suggested implications for practice include interventions to promote adherence to therapeutic regimens, to educate about CF, and to teach problem solving strategies and social skills. Anticipatory guidance about issues faced during middle childhood should be considered when planning the timing of interventions. Coping methods commonly used by children should be considered when planning interventions.

Although implications for further research were not discussed, interventions should undergo evaluation to identify strategies that result in optimal outcomes for children. Caution should be taken in extending these results to all children with chronic illnesses.

Helene Lacroix, RN, MSc Paediatric Manager Victorian Order of Nurses Toronto, Ontario, Canada

1 Mulderij KJ. Child Care Health Dev 1997; 23:379-89. 